\title{
Flux Motion Studies by Means of Temperature Measurement in Magnetizing Processes for HTSC Bulks
}

\author{
Hiroyuki Fujishiro, Tetsuo Oka, Kazuya Yokoyama, Masahiko Kaneyama, and Koshichi Noto
}

\begin{abstract}
The temperature rises $\Delta T(t)$ of cryo-cooled Sm$\mathrm{BaCuO}$ bulk superconductor have been measured after applying the pulse magnetic field from $B_{\text {ex }}=3.01$ to $5.42 \mathrm{~T}$ for various initial stage temperatures $\left(T_{s}=40 \mathrm{~K} \sim 70 \mathrm{~K}\right)$. For each $T_{\mathrm{s}}$ and $B_{\text {ex }}$, the observed systematic change of $\Delta T$ and the trapped magnetic field $B_{T}$ can be explained in terms of the diagram of the maximum temperature measured at the bulk center surface $\left(T 0_{\max }\right)$ vs. $B_{\mathrm{T}}$. The maximum temperature rise $\Delta T_{\max }$ decreases with increasing $T_{\mathrm{s}}$ under the identical $B_{\text {ex }}$.
\end{abstract}

Index Terms-High $T_{\mathrm{c}}$ bulk superconductors, pinning and viscous loss, pulse field magnetizing, temperature measurement.

\section{INTRODUCTION}

$\mathbf{F}$ OR the practical applications of the melt-processed RE$\mathrm{BaCuO}$ superconductors (RE: rare earth ions), the pulse field magnetizing (PFM) have been intensively investigated and developed as well as the static field-cooled magnetizing (FCM) [1]. It is necessary to realize a wider space with higher magnetic field for the applications such as a magnetic separation. For this purpose, new proposals using large-size bulk crystals (e.g., $\sim 100 \mathrm{~mm} \phi$ ) [2] or the assembly of the several small-size bulks [3] have been made. In case of magnetizing these bulks, it is expensive and is not functional to use a superconducting magnet with a large bore, in which these bulks are to be inserted. The PFM has a promising potential to solve these difficulties because of the relatively compact and inexpensive setup. However, the trapped magnetic field $\left(B_{\mathrm{T}}\right)$ in bulks by PFM is currently lower than that by $\mathrm{FCM}\left(B_{\mathrm{T}}(\mathrm{FC})\right)$ below the liquid nitrogen temperature [4]. The main cause has been attributed to the temperature rise $\Delta T$ due to the dynamical motion of the magnetic fluxes against the vortex pinning force $F_{\mathrm{p}}$ and the viscous force $F_{\mathrm{v}}$. However, there have been few systematic experimental studies to relate $\Delta T$ with the $B_{\mathrm{T}}$ value. We investigated

Manuscript received October 20, 2003. This work was supported in part by Japan Science and Technology Corporation under the Joint-Research Project for Regional Intensive in the Iwate Prefecture on "Development of practical applications of magnetic field technology for use in the region and in everyday living."

H. Fujishiro and M. Kaneyama are with the Faculty of Engineering, Iwate University, Morioka, 020-8551 Japan (e-mail: fujishiro@iwate-u.ac.jp; 2203004@iwate-u.ac.jp).

T. Oka and K. Yokoyama are with the Iwate Industrial Promotion Center, Morioka 020-0852, Japan (e-mail: toka@iwate-techno.com; yoko@iwate-techno.com).

K. Noto was with the Faculty of Engineering, Iwate University. He is now with Iwate Industrial Promotion Center, Morioka 020-0852, Japan (e-mail: notok@abox2.so-net.ne.jp).

Digital Object Identifier 10.1 109/TASC.2004.830392 the time evolution and spatial distribution of $\Delta T(t)$ on the surface of the cryo-cooled $\mathrm{YBaCuO}$ [5] and $\mathrm{SmBaCuO}$ [6] bulk superconductors after applying the pulse fields, where the initial stage temperature $T_{\mathrm{s}}$ was maintained in $38 \mathrm{~K}$. It has been found that heat is mainly generated in the peripheral region of the bulk disk and diffuses toward the central region. The magnetic fluxes have been confirmed to move more easily through the growth sector regions (GSR's) than along the growth sector boundaries (GSB's). These results were consistent with the distribution of $B_{\mathbf{T}}$.

In this paper, we investigate the time and position dependence of $\Delta T(t)$ at various $T_{\mathrm{s}}(40 \mathrm{~K} \sim 70 \mathrm{~K})$ after applying the pulse magnetic field from $B_{\mathrm{ex}}=3.01$ to $5.42 \mathrm{~T}$ for the cryo-cooled $\mathrm{SmBaCuO}$ bulk superconductor. Since both $F_{\mathrm{p}}$ and $F_{\mathrm{v}}$ values depend on $T_{\mathrm{s}}$ and $B_{\mathrm{ex}}$, the $\Delta T(t)$ measurements are expected to offer the important clues to enhance $B_{\mathbf{T}}$.

\section{EXPERIMENTAL PROCEDURE}

A highly c-axis oriented $\mathrm{SmBaCuO}$ bulk superconductor of disk shape with $46 \mathrm{~mm}$ in diameter and $15 \mathrm{~mm}$ in thickness was fabricated by Dowa Mining Co., Ltd, which consisted of 4 growth sectors (GSR1-GSR4) [6]. The bulk was composed of $\mathrm{SmBa}_{2} \mathrm{Cu}_{3} \mathrm{O}_{\mathrm{y}}(\mathrm{Sm} 123)$ and $\mathrm{Sm}_{2} \mathrm{BaCuO}_{5}(\mathrm{Sm} 211)$ with the molar ratio of $\operatorname{Sm} 123: \mathrm{Sm} 211=1.0: 0.3,15.0 \mathrm{wt} \% \mathrm{Ag}_{2} \mathrm{O}$ powder and $0.5 \mathrm{wt} \% \mathrm{Pt}$ powder. The bulk was uniformly impregnated by epoxy resin in vacuum and the epoxy resin layer on both upper and bottom sides of the bulk disk was removed in order to measure the precise temperature on the bulk surface and to reduce the thermal contact resistance against the cold stage. Fig. 1 shows the positions of the temperature measurements on the bulk, which was tightly stuck on the cold stage of a Gifford McMahon (GM) cycle helium refrigerator (AISIN, GR103). The initial stage temperature $T_{\mathrm{s}}$ was changed from $40 \mathrm{~K}$ to $70 \mathrm{~K}$. The temperatures, $T 0$ at the center of the bulk (P0) and, $T 1-T 4$ at P1-P4 in the four GSR's were measured by chromel-constantan thermocouples (76 $\mathrm{m}$ in diameter) adhered to the upper bulk surface using GE7031 varnish. P1 P4 were situated on the central lines of each GSR $9 \mathrm{~mm}$ apart from P0. Each temperature was measured about 7 times/s just after applying the pulse field.

The bulk crystal was magnetized using a pulse coil dipped in liquid $\mathrm{N}_{2}$. The rise time of the pulse field $B_{\mathrm{ex}}$ was about $\sim 10 \mathrm{~ms}$. The strength of $B_{\text {ex }}$ was determined from the current flowing through the coil, which ranged from $3.01 \mathrm{~T}$ to $5.42 \mathrm{~T}$. The Hall sensor (F.W. Bell, model BHT-921) was adhered to the 


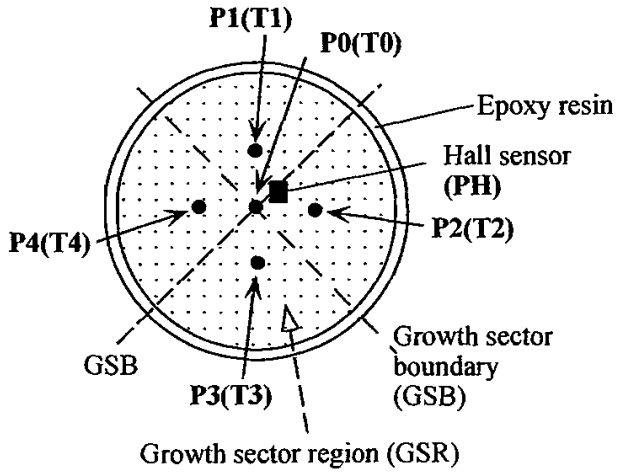

Fig. 1. The positions for the temperature and trapped field measurements on the surface of the $\mathrm{SmBaCuO}$ bulk. P0-P4 for the temperature measurement of $T 0-T 4$ are indicated. The trapped fields $B_{\mathrm{T}}^{3} \mathrm{~mm}_{0}-B_{\mathrm{T}}^{3} \mathrm{~mm}_{4}$ are also measured at $3 \mathrm{~mm}$ just above P0-P4 by a scanning Hall sensor. The trapped field $B_{\mathrm{T}}$ on the bulk surface is measured using a fixed Hall sensor at $\mathrm{PH}$.

position $\mathrm{PH}, 2.5 \mathrm{~mm}$ apart from $\mathrm{P0}$ as shown in Fig. 1, which monitored the $B_{\mathrm{T}}$ value. The two-dimensional distribution of the trapped magnetic field $B_{\mathrm{T}}^{3} \mathrm{~mm}$ was monitored using an axial type Hall sensor, which scanned $3 \mathrm{~mm}$ above the bulk surface stepwise with a pitch of $1.2 \mathrm{~mm}$. The $B_{\mathrm{T}}^{3 \mathrm{~mm}}$ values at P0 P4 $\left(B_{\mathrm{T}}^{3} \mathrm{~mm}_{0} \sim B_{\mathrm{T}}^{3} \mathrm{~mm}_{4}\right)$ were extracted from the distribution. The trapped field $B_{\mathrm{T}}$ (FC) by FCM was also measured at several temperatures using a cryo-cooled superconducting magnet. During FCM, the static magnetic field of $5 \mathrm{~T}$ was decreased down to $0 \mathrm{~T}$ in $18 \mathrm{~min}$.

\section{RESULTS AND DISCUSSION}

Figs. 2(a) and 2(b) show the time evolutions of temperatures $T 0(t) \sim T 4(t)$ for $\mathrm{P} 0 \sim \mathrm{P} 4$ at $T_{\mathrm{s}}=40 \mathrm{~K}$ after applying the pulse field of $3.01 \mathrm{~T}$ and $4.64 \mathrm{~T}$, respectively. Figs. 2(c) and (d) show $T 0(t) \sim T 4(t)$ at $T_{\mathrm{s}}=50 \mathrm{~K}$ after applying the pulse field of $3.01 \mathrm{~T}$ and $4.64 \mathrm{~T}$. The insets of each figure present the $B_{\mathrm{T}}^{3} \mathrm{~mm}$ values at $\mathrm{P} 0 \sim \mathrm{P} 4$. It can be seen that the maximum temperature rise $\Delta T_{\max }$, the time evolution of temperature, and the $B_{\mathrm{T}}^{3 \mathrm{~mm}}$ value for each position change depending on the $T_{\mathrm{s}}$ and $B_{\mathrm{ex}}$ values. In Fig. 2(a) for $B_{\text {ex }}=3.01 \mathrm{~T}$ at $T_{\mathrm{s}}=40 \mathrm{~K}, T 2(t)$ and $T 3(t)$ rise up first with $\Delta T_{\max } \sim 8 \mathrm{~K}$, followed by $T 4(t)$ and $T 1(t)$, and $T 0(t)$ rise up latest. $T 2(t)$ and $T 3(t)$ show a characteristic peak at $t=1.8 \mathrm{~s}$, but the others increase moderately and then reach a maximum at $t=5 \sim 10 \mathrm{~s}\left(\Delta T_{\max } \sim 5 \mathrm{~K}\right)$. All the temperatures return to the initial ones in about $10 \mathrm{~min}$ utes. The rapid and large rise of $T 2$ and $T 3$ with a characteristic peak means that the powerful heat source is located in the very vicinity of $\mathrm{P} 2$ and $\mathrm{P} 3$. Since the flux motion necessarily generates heat inside the bulk through the pinning power loss $P_{\mathrm{p}}$ and the viscous flow power loss $P_{\mathrm{v}}$, such a rapid rise suggests the existence of the easy path for the flux motion due to relatively weaker pinning force. $F_{\mathrm{p}}$ of GSR2 and GSR3. In Fig. 2(b), $T 2(t), T 3(t)$ and $T 4(t)$ rise up faster with a clear peak $\left(\Delta T_{\max } \sim 33 \mathrm{~K}\right)$, followed by $T 1(t)$, and $T 0(t)$ rise up latest $\left(\Delta T_{\max } \sim 28 \mathrm{~K}\right) . \Delta T_{\max }$ increases with increasing $B_{\text {ex }}$ and the spatial distribution of the generated heat depends on $B_{\text {ex }}$.

In Fig. 2(c) for $B_{\mathrm{ex}}=3.01 \mathrm{~T}$ at $T_{\mathrm{s}}=50 \mathrm{~K}, T 2(t), T 3(t)$ and $T 4(t)$ rise up first with a clear peak at $t=1.8 \mathrm{~s}$, followed by $T 1(t)$, and then $T 0(t)$ rise up latest. $\Delta T_{\max }$ is $\sim 8 \mathrm{~K}$ for $T 2, T 3$ and $T 4$, which is similar to that for $B_{\mathrm{ex}}=3.01 \mathrm{~T}$ at $T_{\mathrm{s}}=40 \mathrm{~K}$ shown in Fig. 2(a). In Fig. 2(d), on the other hand, $T(t)$ 's for P1 P4 rise up simultaneously and steeply and do not show a clear peak. $\Delta T_{\max }$ is $\sim 22 \mathrm{~K}$ and slightly smaller than those for $B_{\text {ex }}=4.64 \mathrm{~T}$ at $T_{\mathrm{s}}=40 \mathrm{~K}$ shown in Fig. 2(b). The rapid rise in $T 1-T 4$ means that the fluxes penetrate pretty uniformly through the surfaces of the circumference of the 4 GSR's. In all the cases of the flux penetration from $T_{s}=40 \mathrm{~K}$ to $70 \mathrm{~K}, T 0(t)$ at the center of the bulk rise up latest, because the main heat generation always takes place in the peripheral region where the magnetic fluxes move faster.

The distribution of $B_{\mathrm{T}}^{3 \mathrm{~mm}}$ also changes depending on $T_{\mathrm{s}}$ and $B_{\text {ex }}$. In the inset of Fig. $2(\mathrm{a})$ for $B_{\mathrm{ex}}=3.01 \mathrm{~T}$ at $40 \mathrm{~K}, B_{\mathrm{T}}^{3} \mathrm{~mm}$ is larger for $\mathrm{P} 2\left(B_{\mathrm{T}}^{3} \mathrm{~mm}_{2}=0.8 \mathrm{~T}\right)$ and $\mathrm{P} 3\left(B_{\mathrm{T}}^{3} \mathrm{~mm}_{3}=0.7 \mathrm{~T}\right)$, where $\Delta T_{\max }$ is also larger. However, $B_{\mathrm{T}}^{3 \mathrm{~mm}_{1}}$ and $B_{\mathrm{T}}^{3} \mathrm{~mm}_{4}$ are quite small and $\Delta T_{\max } 1$ and $\Delta T_{\max } 4$ are small as well. The behaviors of $B_{\mathrm{T}}^{3 \mathrm{~mm}}$ and $T 0(t) \sim T 4(t)$ suggest that the surface barrier against the flux penetration has been destroyed in GSR2 and GSR3 for $B_{\text {ex }}=3.01 \mathrm{~T}$. On the other hand, the barriers are kept intact in GSR1 and GSR4. In the inset of Fig. 2(b) for $B_{\text {ex }}=4.64 \mathrm{~T}$ at $40 \mathrm{~K}, B_{\mathrm{T}}^{3} \mathrm{~mm}$ is the largest for the GSR1 and the smallest for the GSR3 with a V-shaped distribution, making a contrast to $B_{\mathrm{ex}}=3.01 \mathrm{~T}$. This result is consistent with the existence of hard path through $\mathrm{P} 1$ and the easy path through P3 for the flux motion. In the higher $T_{\mathrm{s}}(=50 \mathrm{~K})$, the behaviors of $B_{\mathrm{T}}^{3} \mathrm{~mm}$ change. In the inset of Fig. 2(c) for $B_{\mathrm{ex}}=3.01 \mathrm{~T}$ at $50 \mathrm{~K}, B_{\mathrm{T}}^{3 \mathrm{~mm}_{2}}$ and $B_{\mathrm{T}}^{3 \mathrm{~mm}_{3}} 3$ take similar values to those for $B_{\text {ex }}=3.01 \mathrm{~T}$ at $40 \mathrm{~K}$. However, $B_{\mathrm{T}}^{3} \mathrm{~mm}_{4}$ increases from $0.36 \mathrm{~T}$ $(40 \mathrm{~K})$ to $0.88 \mathrm{~T}(50 \mathrm{~K})$. This result indicates that the surface barrier against the flux penetration is destroyed at $50 \mathrm{~K}$ also in GSR4 and that $B_{\mathrm{T}}$ and $\Delta T_{\max }$ increase, resultantly. In the inset of Fig. 2(d), the $B_{\mathrm{T}}^{3 \mathrm{~mm}}$ values decrease at P1, P2, and P4, and the V-shaped $B_{\mathrm{T}}^{3 \mathrm{~mm}}$ distribution is more broadened compared with those for $B_{\mathrm{ex}}=4.64 \mathrm{~T}$ at $40 \mathrm{~K}$.

We define the rise time $t(60 \%)$ to reach $60 \%$ of the maximum temperature rise $\Delta T_{\max }$. Figs. $3(\mathrm{a})$ and $3(\mathrm{~b})$ show $t(60 \%)$ as a function of $T_{\mathrm{s}}$ for $B_{\mathrm{ex}}=3.01$ and $4.64 \mathrm{~T}$. In Fig. 3(a), $t(60 \%)$ for $T 2$ and $T 3$ is smaller at $T_{\mathrm{s}}=40 \mathrm{~K}$ because of the destruction of the surface barrier. $t(60 \%)$ for $T 4$ and $T 1$ become short at $T_{\mathrm{s}}=50 \mathrm{~K}$ and $60 \mathrm{~K}$, respectively, for the same reason. All the surface barriers in the circumference region against the flux penetration are destroyed at $T_{\mathrm{s}} \geq 60 \mathrm{~K}$ for $B_{\text {ex }}=3.01 \mathrm{~T}$. These results are caused by the decrease of $F_{\mathrm{p}}$ with the increase in $T_{\mathrm{s}}$. The $t(60 \%)$ value is determined by the thermal diffusivity $\alpha(T)$ of the bulk and the distance between the measuring point and the heat source. $\alpha(T)$ decreases with increasing $T$ ( $\alpha=23.0$ and $8.2 \mathrm{~mm}^{2} / \mathrm{s}$ at $40 \mathrm{~K}$ and $70 \mathrm{~K}$, respectively) [7]. Accordingly, the decrease of $t(60 \%)$ at $\mathrm{P} 4$ and $\mathrm{P} 1$ with increasing $T_{\mathrm{s}}$ does not come from $\alpha(T)$, but originates from the approach of the heat source to P4 and P1. In Fig. 3(b) for $B_{\text {ex }}=4.64 \mathrm{~T}, t(60 \%)$ for $\mathrm{P} 1 \sim \mathrm{P} 4$ is $0.9 \sim 1.2 \mathrm{~s}$ and independent of $T_{\mathrm{s}}$ and the position. These results are consistent with the uniform flux intrusion and heat generation. On the other hand, $t(60 \%)$ at $\mathrm{P} 0$ shows a large value $(2.4 \sim 3.5 \mathrm{~s})$ and monotonically increases with increasing $T_{\mathrm{s}}$, which is attributable to the decrease of $\alpha(T)$.

Fig. 4 shows $\Delta T 0_{\max }$ at $\mathrm{PO}$ for each $T_{\mathrm{s}}$ as a function of $B_{\text {ex }}$. The inset presents the $B_{\text {ex }}$ dependence of $B_{\mathrm{T}}$ monitored by the Hall sensor fixed at PH shown in Fig. 1. (note that P0 

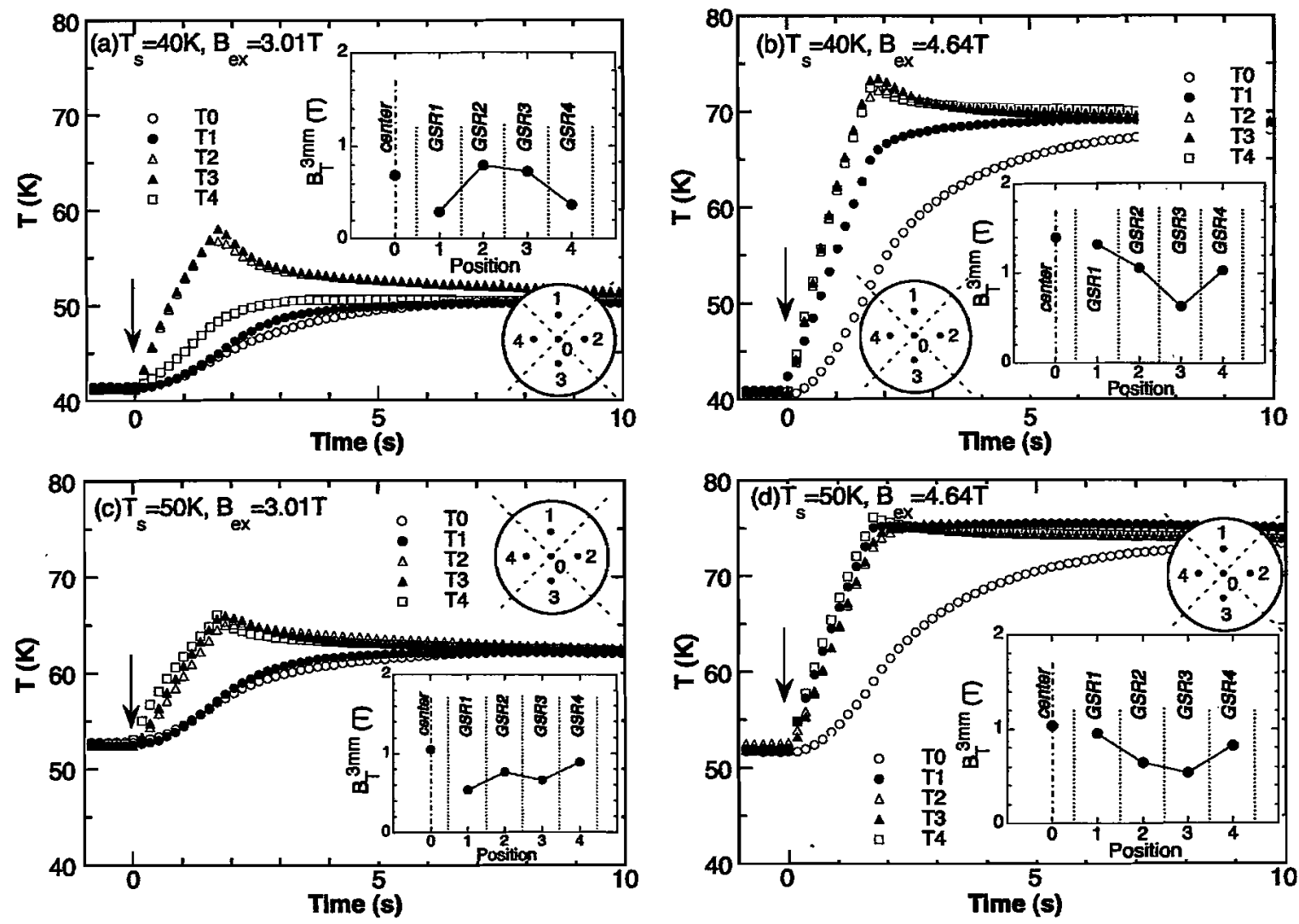

Fig. 2. The time evolution of temperatures $T 0(t) \sim T 4(t)$ for P0 P4 at $T_{\mathrm{s}}=40 \mathrm{~K}$ after applying the pulse field of (a) $3.01 \mathrm{~T}$ and (b) $4.64 \mathrm{~T}$, respectively. (c) and (d) show $T 0(t) \sim T 4(t)$ at $T_{\mathrm{s}}=50 \mathrm{~K}$ after applying the same pulse fields as (a) and (b), respectively. The insets of figures present the $B_{\mathrm{T}}^{3} \mathrm{~mm}$. values at each position.

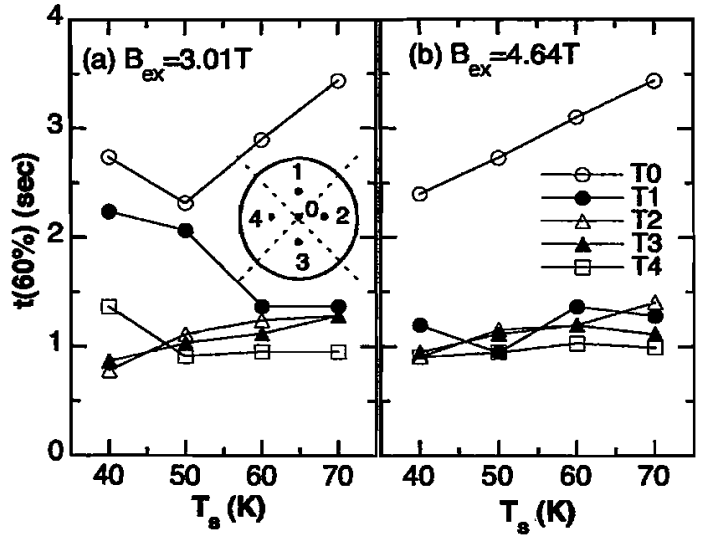

Fig. 3. The $t(60 \%)$ value at the positions (P0 P4) as a function of the initial stage temperature $T_{\mathrm{s}}$ for (a) $B_{\mathrm{ex}}=3.01$ and (b) $4.64 \mathrm{~T}$.

is the nearest position to $\mathrm{PH}$ ). For each $T_{\mathrm{s}}, \Delta T 0_{\max }$ increases and then shows a saturation tendency with increasing $B_{\text {ex. }}$. The $\Delta T 0_{\max }$ values are unexpectedly almost identical for each $T_{\mathrm{s}}$ at $B_{\text {ex }}=3.01 \mathrm{~T}$, but increases with decreasing $T_{\mathrm{s}}$, especially for higher $B_{\mathrm{ex}}$. These results can be explained by the decrease of $F_{\mathrm{p}}$ and by the increase of the specific heat $C$ at higher temperature. In the inset of Fig. 4 , at $T_{\mathrm{s}}=40 \sim 60 \mathrm{~K}, B_{\mathrm{T}}$ increases for $B_{\mathrm{ex}}=3.87 \mathrm{~T}$ in comparison to $B_{\mathrm{ex}}=3.01 \mathrm{~T}$, takes a maximum, and decreases with further increase of $B_{\mathrm{ex}} . B_{\mathrm{T}}$ at $3.01 \mathrm{~T}$ increases with increasing $T_{\mathrm{s}}$ up to $60 \mathrm{~K}$ and then decreases. On the other hand, $B_{\mathrm{T}}$ at $5.42 \mathrm{~T}$ monotonically decreases with increasing $T_{\mathrm{s}}$.

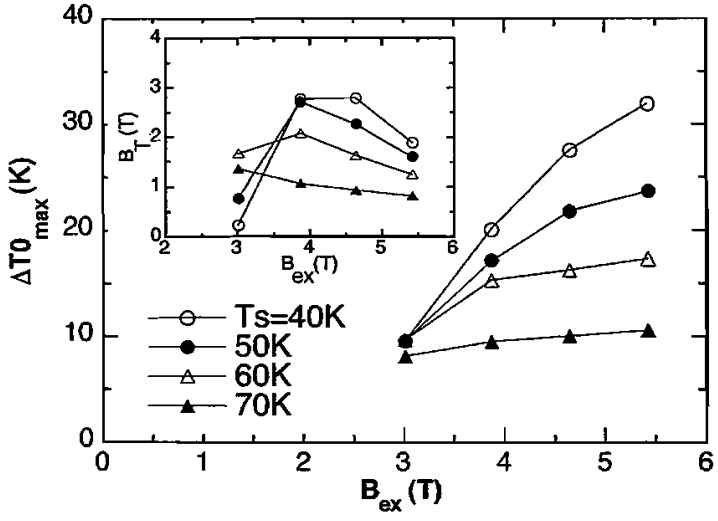

Fig. 4. The maximum temperature rise $\Delta T 0_{\max }$ at $\mathrm{PO}$ for each $T_{\mathrm{s}}$ as a function of $B_{\text {ex }}$. The inset presents the $B_{\text {ex }}$ dependence of $B_{\mathrm{T}}$ monitored by the Hall sensor fixed at PH shown in Fig. 1.

Fig. 5 shows the summary of $B_{\mathrm{T}}$ vs. $T 0_{\max }$ in this study. For each $T_{\mathrm{s}}$, four data sets of $\left(B_{\mathrm{T}}, T 0_{\max }\right)$ are plotted for $B_{\mathrm{ex}}=$ $3.01,3.84,4.64$ and $5.42 \mathrm{~T}$. The measured trapped field $B_{\mathrm{T}}(\mathrm{FC})$ by FCM corresponding to the temperature $T 0_{\max }$ is also presented. In the case of $T_{\mathrm{s}}=40 \mathrm{~K}$, the two data sets of $\left(B_{\mathrm{T}}\right.$, $\left.T 0_{\max }\right)$ are situated below the $B_{\mathrm{T}}(\mathrm{FC})-T 0_{\max }$ line for $B_{\mathrm{ex}} \leq$ $3.82 \mathrm{~T}$. For $B_{\mathrm{ex}} \geq 4.64 \mathrm{~T}, T 0_{\max }$ touches the line owing to the large temperature rise and then $B_{\mathrm{T}}$ decreases following the line. In the case of $T_{\mathrm{s}}=50 \mathrm{~K}$, only the data set for $B_{\mathrm{ex}}=3.01 \mathrm{~T}$ is situated below the $B_{\mathrm{T}}(\mathrm{FC})-T 0_{\max }$ line, and for $B_{\mathrm{ex}} \geq 4.64 \mathrm{~T}$, $T 0_{\max }$ touches the line and the $B_{\mathrm{T}}$ decreases. On the other hand, for $T_{\mathrm{s}}=60$ and $70 \mathrm{~K}$, all the data sets touch the line already at 


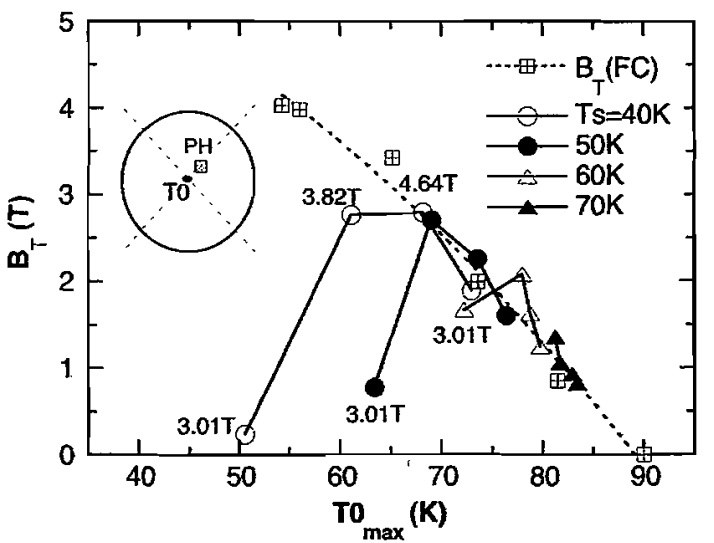

Fig. 5. The summary of the trapped field $B_{\mathrm{T}}$ at $\mathrm{PH}$ vs. the maximum temperature $T 0_{\max }$ at $\mathrm{PO}$. For each $T_{\mathrm{s}}$, four data sets of $\left(B_{\mathrm{T}}, T 0_{\max }\right)$ are plotted for $B_{\text {ex }}=3.01,3.84,4.64$ and $5.42 \mathrm{~T}$. The trapped field $B_{\mathrm{T}}(\mathrm{FC})$ by FCM is also shown.

$B_{\text {ex }}=3.01 \mathrm{~T}$ and $B_{\mathrm{T}}$ monotonically decreases with increasing $B_{\text {ex }}$. These results demonstrate that the flux trapping ability by the PFM technique can be systematically explained as limited by the $B_{\mathrm{T}}$ (FC) $-T 0_{\max }$ line [6].

In summary, we measured the temperature rises $\Delta T(t)$ of a cryo-cooled SmBaCuO bulk superconductor after applying the pulse magnetic fields ( $\sim 10 \mathrm{~ms}$ of the rise time) from $B_{\mathrm{ex}}=3.01$ to $5.42 \mathrm{~T}$ for various initial stage temperatures $\left(T_{\mathrm{s}}=40 \sim\right.$ $70 \mathrm{~K}$ ). The maximum temperature rise $\Delta T_{\max }$, the time evolution of temperature $T 0(t) \sim T 4(t)$, and the trapped field $B_{\mathrm{T}}^{3 \mathrm{~mm}}$ value for each position changes depending on the $T_{\mathrm{s}}$ and $B_{\text {ex }}$ values. When $T_{s}$ increases, $\Delta T_{\max }$ decreases due to the reduction of the pinning force $F_{\mathrm{p}}$ and the specific heat as shown in Fig. 4. As a result, the magnetic fluxes easily penetrate into the bulk and can be trapped at higher $T_{\mathrm{s}}$ even for relatively lower $B_{\mathrm{ex}}$ value; $B_{\mathrm{T}}$ increases with increasing $T_{\mathrm{s}}$ for $B_{\mathrm{ex}}=3.01 \mathrm{~T}$, as shown in the inset of Fig. 4. However, at higher $T_{\mathrm{s}}$, there is only small temperature margin between $T_{\mathrm{s}}$ and the superconducting transition temperature $T_{c}(=93 \mathrm{~K})$ and the flux trapping ability of the bulk necessarily decreases. In this sense, $T_{\mathrm{s}}$ must be decreased as low as possible in order to obtain the higher $B_{\mathrm{T}}$ values. The $B_{\mathrm{T}}$ behavior at the various $T_{\mathrm{s}}$ and for various $B_{\mathrm{ex}}$ can be clearly understood on the basis of the $B_{\mathrm{T}}(\mathrm{FC})-T 0_{\max }$ line, which corresponds to the maximum-trapped field of the bulk.

The temperature rise $\Delta T$ is caused by both the pinning power loss $P_{\mathrm{p}}$ and the viscous flow power loss $P_{\mathrm{v}}$. Which power loss is dominant for $\Delta T$ in the case of each $T_{\mathrm{s}}$ and $B_{\mathrm{ex}}$ is open to question. In order to clarify this problem and to improve the PFM technique for the $B_{\mathrm{T}}$ enhancement, it is necessary to analyze the data more closely based on the generated heat $Q$ for various $T_{\mathrm{s}}$ and $B_{\mathrm{ex}}$.

\section{ACKNOWLEDGMENT}

The authors greatly thank Prof. M. Ikebe of Iwate University for valuable suggestions.

\section{REFERENCES}

[1] U. Mizutani, T. Oka, Y. Itoh, Y. Yanagi, M. Yoshikawa, and H. Ikuta, "Pulse-field magnetization applied to high- $T_{c}$ superconductors," IEEE Trans. Appl. Supercond., vol. 6, pp. 235-246, 1998.

[2] T. Fujimoto, M. Morita, N. Masahashi, and T. Kaneko, "Fabrication of $100 \mathrm{~mm}$-diameter $\mathrm{Y}$-Ba-Cu-O bulk QMG superconductors with large levitation forces," in Inst. Phys. Conf. Ser, vol. 167, 2000, pp. 79-83.

[3] T. Oka, K. Yokoyama, and K. Noto, "Construction of a strong magnetic field generator with wide magnetic poles by bulk superconductors," Physica $C$.

[4] H. Ishihara, H. Ikuta, Y. Itoh, Y. Yanagi, M. Yoshikawa, T. Oka, and U. Mizutani, "Pulsed field magnetization of melt-processed Sm-Ba-Cu-O," Physica C, vol. 357-360, pp. 763-766, 2001.

[5] H. Fujishiro, T. Oka, K. Yokoyama, and K. Noto, "Time evolution and spatial distribution of temperature in YBCO bulk superconductor after pulse field magnetizing," Supercond. Sci. Tech., vol. 16, pp. 809-814, 2003.

[6] —, "Temperature rise in Sm-based bulk superconductor after applying iterative pulse fields," Supercond. Sci. Tech., vol. 17, pp. 51-57, 2004.

[7] H. Fujishiro and S. Kohayashi, "Thermal conductivity, thermal diffusivity and thermoelectric power in Sm-based bulk superconductors," IEEE Trans. Appl. Supercond., vol. 12, no. 1, pp. 1124-1127, March 2002. 